\title{
Influence of Water Activity on Production of Bacillus thuringiensis by Solid State Fermentation
}

\author{
Lúcia Helena Pelizer ${ }^{1}$ and Iracema de Oliveira Moraes ${ }^{2}$ \\ 1. Department of Food Engineering, Instituto de Ciências Tecnológicas e Exatas (ICTE), Universidade Federal do Triângulo \\ Mineiro (UFTM), Av. Doutor Randolfo Borges Júnior, 1250, CEP 38064-200, Uberaba, MG \\ 2. Probiom Tecnologia, R. Latino Coelho, 1301, CEP 13087010, Campinas, SP, Brasil
}

Received: Feburary 16, 2012 / Published: June 20, 2012.

\begin{abstract}
Bacillus thuringiensis can be produced by a fermentative process using agro-industrial residues as substrates. The culture medium in solid state fermentation (SSF) is composed by moist nutrients. The water is a limiting factor in the SSF, differently from that in submerged fermentation. The solid culture medium must have a minimum moisture content to allow microbial growth but the concept of water availability in the substrate is best explained in terms of water activity (Aw). This study investigated the water activity influence on the solid state fermentation of Bacillus thuringiensis using rice husk, bran and grits as substrates. Three culture media were tested: (i) rice grits; (ii) rice grits and rice husk; (iii) rice bran and rice husk. In order to study hygroscopic characteristics of each medium, isotherms were constructed. For each culture medium, two processes were carried out: one with low Aw and the other with high Aw. The fermentations with B. thuringiensis were made in plastic bags containing the culture medium. The results showed differences in productivity. The best results were obtained for the medium composed by rice grits. The productivity ((spores/g medium)/h) for the process using initial Aw of 0.620 was $3.0 \times 10^{4}$ and for the initial Aw of 0.965 was $1.0 \times 10^{6}$.
\end{abstract}

Key words: Bacillus thuringiensis, water activity, solid state fermentation, rice residues.

\section{Introduction}

The use of chemical pesticides in agriculture has caused extensive damage to the environment since their high toxicity is harmful to beneficial insects, domestic animals, and to man. In addition, some species have already shown resistance problems. An alternative to pest control may be the use of pesticides or the rational combination of chemical and biological pesticides. There is a wide variety of microorganisms (fungi, bacteria, and viruses) that can act as entomopathogens in the biological control of pests. Among them we find Bacillus thuringiensis, which is a bacterium that has great potential, is effective against a wide range of insects, and is harmless to humans, animals, beneficial insects, and natural predators [1-5]

Corresponding author: Lúcia Helena Pelizer, Ph.D., professor, research field: bioprocess engineering. E-mail: lucia.pelizer@gmail.com.
B. thuringiensis can be produced by fermentation processes using agro-industrial residues of low commercial value as substrates. Throughout the various stages in rice processing, from harvest to the production of graded, polished white rice, the following by-products and residues are produced: rice straw, husk, bran, and grit, which can be considered residues of great potential use as substrates [6-8].

Ecologically, the bio-utilization of these residues for pesticide production can be considered very important because besides being a solution to industries, these products do not harm the environment and will be used in agriculture, which provides raw materials for their production [9].

Among the techniques used in fermentation processes, the following can be highlighted: fermentation in liquid medium or submerged fermentation (SmF) and solid-state fermentation (SSF). 
In SSF, the culture medium is composed of moist solid materials. The nutrients in this case are heterogeneously distributed. In this technique, the microbial growth occurs in the humid substrate, which is similar to what occurs in the natural habitat of microorganisms [10, 11].

Recently, there has been a resurgence of interest in the SSF techniques, which have mostly been used in Eastern countries for the production of fermented foods and beverages and also as enzymes. This renewed interest is due to the fact that there are some advantages of SSF over SmF, such as lower energy requirements and smaller reactors, and also because the product is obtained in concentrated form. One of the greatest conveniences of the SSF is the use of small amounts of water, which drastically reduces energy consumption during product recovery in addition to producing smaller volume of effluents [7, 11-14].

Zhao et al. [14] studied the fermentation of Bacillus licheniformis in solid state fermentation. A maximum of $1.7 \times 10^{11}$ cells/g dry substrate was obtained using rice straw powder $(300 \mathrm{~g} / \mathrm{kg})$ and wheat bran $(700$ $\mathrm{g} / \mathrm{kg}$ ) supplemented with glucose (40 $\mathrm{g} / \mathrm{kg})$, peptone (20 g/kg), yeast extract (20 g/kg), $\mathrm{KH}_{2} \mathrm{PO}_{4}(10 \mathrm{~g} / \mathrm{kg})$, and $\mathrm{CaO}(5 \mathrm{~g} / \mathrm{kg})$ with an initial moisture content of $65 \%$.

Patel et al. [12] presented a thorough literature review revealing the state of the art of SSF; nevertheless, the focus is on the most recent developments in the last five years in SSF products and processes. The authors made reference to only one paper published in 2005. The manuscript published by Vimala Devi et al. [7] investigated the cost of production of Bacillus thuringiensis. The standard fermentation was made on wheat bran based medium in $250 \mathrm{~mL}$ Erlenmeyer flasks. Scale-up production of B. thuringiensis on the best medium in plastic tubs with aeration at $8 \mathrm{~h}$ intervals starting $16 \mathrm{~h}$ after incubation yielded a significant increase in spore count and toxin content of the product. The material cost for production of $1 \mathrm{~kg}$ of $B$. thuringiensis was approximately 0.7 U.S. dollars. On the other hand, Sella et al. [13] published a manuscript on Bacillus atrophaeus spores' production by solid-state fermentation. The authors used soybean molasses as substrate and sugarcane bagasse as support.

According to the literature, there are few studies on the influence of water activity on solid state fermentation, especially with $B$. thuringiensis. However, in order for microbial growth to occur, in addition to sources of carbon, nitrogen, and some salts, particularly for the SSF, a minimum amount of water required for the development of physiological activity of a microorganism should be taken into account, which varies according to type and variety. Bacteria, for example, require greater amounts of water than fungi and yeasts. Therefore, water in the SSF becomes a limiting factor in this process, which does not occur in the liquid fermentation, in which there is an abundant aqueous phase. In the solid culture medium, minimum moisture content is necessary for microbial growth; however, the concept of water available in the substrate for the use of microorganism is very different from moisture content, and it is better expressed in terms of water activity (Aw). The relationship between substrate moisture and its corresponding water activity at a given temperature is represented by curves called isotherms showing the hygroscopic characteristics of each material [11, 15-20].

The objective of this study is to investigate the influence of water activity (Aw) and hygroscopic characteristics of culture media on production of Bacillus thuringiensis by solid state fermentation.

\section{Material and Methods}

Chemical composition of substrates: moisture [21], ash [21], protein [21], lipid [22], reducing sugars and total sugars [23] were determined.

Particle size analysis: the particle size of each substrate was determined using the methodology developed by the Instituto Adolfo Lutz [23].

Water activity: water activity was measured by the 
graphic interpolation method [24].

Media: the following rice processing industrial residues were used as substrates: (i) rice grits; (ii) ground rice grits and rice husk; and (iii) rice bran and rice husk.

Isotherms: the study of the hygroscopic characteristics of the culture media was conducted by constructing water adsorption isotherms by measuring the water activity (Aw) and moisture content of each culture medium with addition of different amounts of water based on the medium without any addition of water.

Microorganism: The microorganism used was Bacillus thuringiensis, obtained from the commercial biopesticide "Dipel”.

Fermentation: The culture media were placed in polypropylene bags which were sterilized by autoclaving. For each medium, two fermentations were made with different initial values of Aw. The first one corresponded to the culture medium without the addition of water and second with an Awi value around 0.960 (Table 1). The inoculum concentration was $1.0 \times 10^{7}$ cells $/ \mathrm{mL}$, on average, measured using a Neubauer chamber.

Spore count: the fermentation process was monitored by counting the spores of $B$. thuringiensis in the culture medium by the pour plate count method [25].

\section{Results and Discussion}

The chemical composition of the substrates used varied. Table 2 shows that rice bran is richer in proteins and sugars than rice husk and grits. According to Dors et al. [26], the whole grain consists of several tissues with different structure, chemical composition, and functions. The husk weight is about $15 \%$ to $30 \%$ of the grain weight, and mineral (silica) as well as cellulose are the major components of the husk. On the other hand, the bran is composed of layers surrounding the starchy endosperm cells of the rice grain; it is rich in proteins, lipids, vitamins, and minerals constituting $5 \%-7 \%$ of the grain weight.
Table 1 Fermentations with different culture medium compositions.

\begin{tabular}{lll}
\hline Experiment & Culture medium composition & $\begin{array}{l}\text { Initial water } \\
\text { activity (Awi) }\end{array}$ \\
\hline 1 & rice grits & 0.620 \\
2 & rice grits & 0.965 \\
3 & ground rice grits + rice husk & 0.780 \\
4 & ground rice grits + rice husk & 0.962 \\
5 & rice bran + rice husk & 0.615 \\
6 & rice bran + rice husk & 0.969 \\
\hline
\end{tabular}

Table 2 Chemical composition of substrates.

\begin{tabular}{lrrr}
\hline Components (\%) & Grits & Bran & Husk \\
\hline Moisture & 15.44 & 10.47 & 10.14 \\
Ash & 0.53 & 9.94 & 15.53 \\
Protein & 6.87 & 10.83 & 1.96 \\
Lipids & 1.15 & 3.99 & 0.82 \\
Total sugar & 2.87 & 4.56 & 1.07 \\
Reducing sugar & 1.02 & 0.67 & 0.68 \\
\hline
\end{tabular}

Since the husk is the poorest substrate in nutrients, it can be used as an adjuvant in the process, for example, along with fine grained substrates that have a tendency to remain aggregated and thereby assist in the improvement of mass transfer between its particles. This was done in this study when bran and ground rice grits were used. Rice husk could also be used as a support for liquid substrates rich in nutrients.

With regard to size, rice grits are considered medium sized particles, rice bran, husk, and ground grits are considered fine grained particles. Rice grit grains have an average diameter of $1.61 \mathrm{~mm}$, whereas the average diameter of ground grit particles is 0.68 $\mathrm{mm}$; for husk it is $0.70 \mathrm{~mm}$, and for bran it is $0.79 \mathrm{~mm}$.

The water adsorption curves obtained for the three compositions of culture medium studied are illustrated in Fig. 1. Those curves were obtained from the substrates without addition of water and that do not undergo any drying process. The only treatment those substrates underwent, in all cases, was sterilization by autoclaving at $121^{\circ} \mathrm{C}$ for 20 minutes. It can be noted that the substrates have different water activity and moisture content showing that the materials to be used in SSF should be studied taking into account their hygroscopic properties. The differences observed between the curves shows a change in the characteristics 


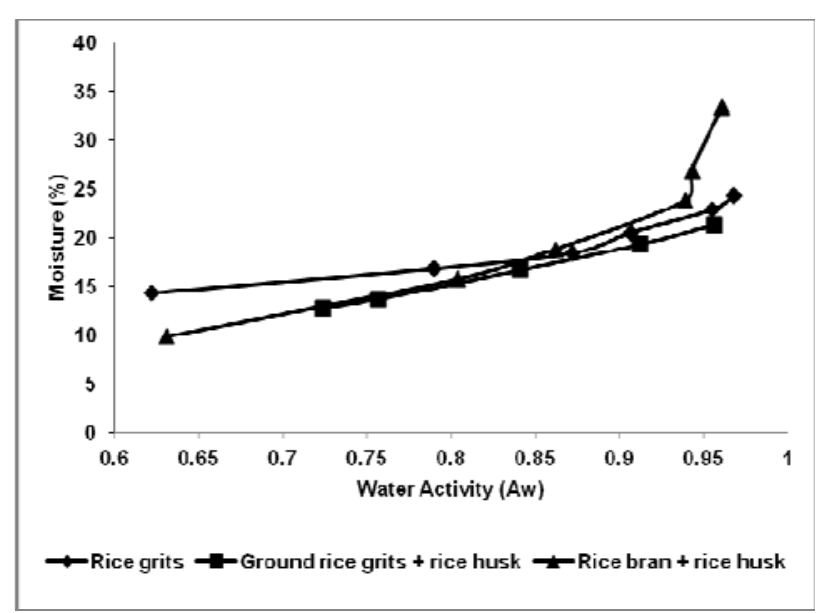

Fig. 1 Culture media water adsorption curves.

of water sorption for each medium studied, which was expected since the substrates have different chemical compositions.

The adsorption curves obtained (Fig. 1) are characterized by two typical regions that describe how the adsorption takes place: for the rice grits, the first region is verified up to an Aw value of 0.9 , and the second region is verified at higher values, where the curve undergoes an abrupt change; for the rice bran and husk, the first region occurs up to an Aw value of 0.95, whereas for rice grits and husk only the first region is observed. The first region, where moisture undergoes slighter variation with increasing Aw, corresponds to the adsorption of additional layers to the monolayer that is formed when there is low moisture and low water activity. Water fraction in this region is therefore less tightly bound, and the enthalpy of vaporization is slightly larger than that of pure water. The second region, characterized by a greater increase in moisture with slighter Aw variations, reflects the material's water condensation followed by dissolution of soluble matter present, i.e., this region represents the actual free water; water is mechanically involved in the open spaces of the system. This second region is quite pronounced for the rice bran and husk. For the ground rice grits and husk, weakly bound water predominates. With regard to rice grits in the first region, the slope of the isotherm is less pronounced than that of the other two substrates, which indicates a smaller amount of water in the outermost layer, but with increasing Aw values, it falls between those of the other two substrates.

When constructing these curves, it was found that to obtain similar Aw values, the amount of water added to each composition of culture medium was not the same. For the rice bran and husk mixture, the required amount of water is twice as much as that for the rice grits to obtain an Aw value of approximately 0.960. This shows once again that if the chemical composition of each substrate is different, their hygroscopic characteristics will also be different.

The Aw value also indicates the potential of water that is directly connected with the energetic state of water in organic substrates. The water potential of a solid substrate is essentially defined as the sum of two components: osmotic potential (related to the concentration and physicochemical properties of solutes) and matric potential (related to adsorption and capillarity of the solid matrix). For the equilibrium between the liquid, solid, and gaseous phases, water potential may be related to relative humidity $(\mathrm{RH})$ of the gas phase or the Aw of the substrate [11, 16-18, 27, 28].

According to Murthy et al. [11], water activity (Aw) sets the amount of unbound water available in the immediate surroundings of the microorganism; therefore it better defines the limits of growth of microorganisms because they do not recognize the moisture content of the material, but rather the amount of water available, whose variation depends on the solute. In solid state culture, microbial cells grow either on the surface or within the particle of solid matrix, where water is sufficient to sustain growth [29].

Until recently, Aw was considered as a physicochemical parameter in two scientific disciplines: physical chemistry and food microbiology. According to the former, it was considered the thermodynamic concentration of water; and according to the latter, it was considered the growth limit of food spoiling microorganisms. The reason why water activity is used instead of moisture content in 
microbiology is that it has been shown that microorganisms which deteriorate food can grow in a wide range of moisture levels [30]. Hahn-Hägerdal [18] stated that Aw should be seen as another parameter of the physicochemical process (such as $\mathrm{pH}$, $\mathrm{PO}_{2}$, and temperature), which under certain conditions act synergistically with other parameters, and in some cases can determine the progress of some biological, microbiological, or enzymatic processes.

In the SSF, water should be available for microbial growth and biochemical activities to occur. Every microorganism has a minimum Aw to carry out its metabolic activities. For example, the optimum Aw for fungi is around 0.7 , for yeast it is 0.8 , and for bacteria it is 0.9 [31]. A small variation in these optimum values causes major disturbance in the growth and metabolism of microorganisms [32].

Corona et al. [33] carried out several studies on Aw and microbial growth. For example, in Penicillium roqueforti, Aw is the critical variable for growth and spore production in SSF. Some studies report that the optimum Aw for growth of Trichoderma viride ranges between 0.99 and 0.992, while spore production is maximized at Aw 0.98. On the other hand, the optimum Aw for cyclopeptide production in Metarhizium anisopliae is 0.921 although variations according to media composition were observed. Results found for Gibberella fujikuroi growth rates at different Aw levels minimal values indicated that the minimal Aw value that supported growth was approximately 0.9. Growth rate increased continuously up to an Aw value of 0.995, and then it decreased slowly. The optimum growth for Gibberella fujikuroi was obtained between Aw values of 0.985 and 0.995 .

The induction of sporulation of some genera of fungi and bacteria occurs in response to several factors including: availability of nutrients, unfavorable $\mathrm{pH}$, and osmotic stress, for example, changes in the Aw environment. Bacterial endospores and some types of fungal spores are characterized by the need for special conditions in order to start germination and development. Among those needs, according to Beuchat [31], are optimal values of Aw. Grajek and Gervais [17] found that Aw strongly affects the sporogenesis of Trichoderma viride. Their results proved that the Aw can be a limiting parameter in the culture development.

Lower water activity result in lower mass transfer rates and little water available for microorganisms, and it may be responsible for incomplete conversion of substrate to biomass [11]. However, the behavior of isotherms provides important information regarding the greater or lesser water availability within the range studied (0.6 to 0.9). It is evident that the free water, represented by the second region of the isotherms, will be more readily available, whereas for smaller Aw values, represented by the first region of the isotherms, the water will become available as the layers are used and change the adsorption thermodynamic balance, i.e., the water release rate will be lower. This can be beneficial for microorganism growth.

Fig. 2 shows the growth curves of $B$. thuringiensis based on spore production.

Based on the isotherms, it can be said that in experiments 3 and 4 (performed with ground rice grits and rice husk) the speed of the initial production of

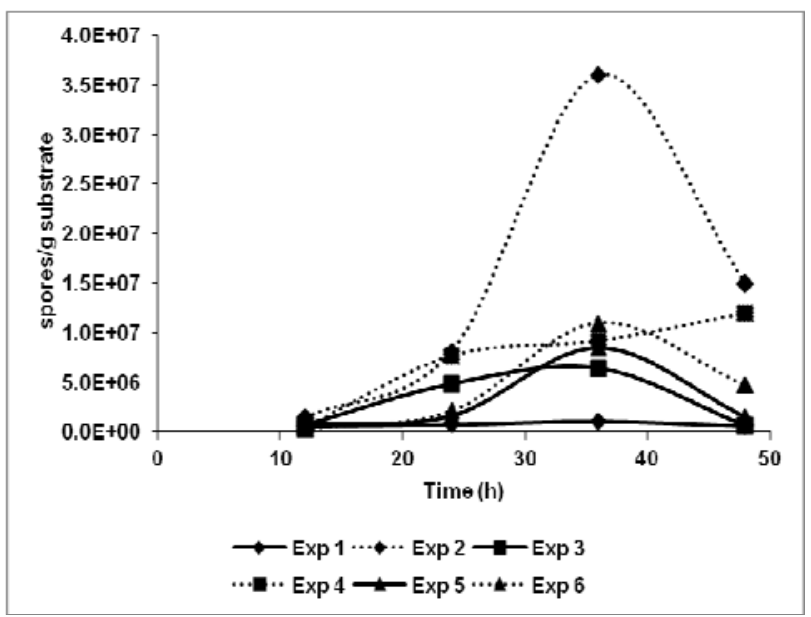

Fig. 2 Growth curves of $B$. thuringiensis in the six experiments performed.

Exp 1: rice grits; Awi: 0.620. Exp 2: rice grits; Awi: 0.965. Exp 3: ground rice grits + rice husk; Awi: 0.780. Exp 4: ground rice grits and rice husk; Awi: 0.962. Exp 5: rice bran + rice husk; Awi: 0.615. Exp 6: rice bran + rice husk; Awi: 0.969. 
spores is greater than that of the experiments with higher amount of free water available. Experiment 2 showed the greatest spore formation $\left(3.5 \times 10^{7}\right.$ spores/g substrate), probably due to the lower water release rate. Except for experiment 4, all experiments had the maximum production of spores in 35 hours.

In general, it can be said that the maximum production of spores is higher in fermentations with higher initial Aw. The greatest difference is in the fermentation of the medium composed of rice grits. In this case, the yield ((number of spores/g medium)/h) for the medium with initial Aw of 0.620 was $3.0 \times 10^{4}$; and for the medium with initial Aw of 0.965, it was $1.0 \times 10^{6}$ (Fig. 1). This can be justified by observing the variation of Aw during fermentation. Despite its increased value in the early hours, the Aw was below 0.900 , except for the greatest production. This may have inhibited the $B$. thuringiensis growth.

In the fermentation with ground rice grits mixed with rice husk as substrates, a small difference in growth was observed, especially in the early hours. While the productivity ((number of spores/g medium $/ \mathrm{h}$ ) in the medium with initial water activity of 0.708 was $1.8 \times 10^{5}$, for the medium with initial Aw of 0.962 , it was $2.6 \times 10^{5}$. This can be explained because although the Aw of the medium with low initial water activity reached lower values than the previous case, it is worth mentioning that the initial Aw of this medium is considerably higher, which could have favored the sporulation of $B$. thuringiensis. On the other hand, in the fermentation with rice bran mixed with rice husk as substrates, the difference in productivity was even lower. For the medium with initial water activity of 0.615 , productivity ((in spores/g medium)/h) was $3.0 \times 10^{5}$, and for the medium with initial Aw of 0.969 , it was $2.6 \times 10^{5}$. This slight difference can be due to the fact that, during fermentation, the Aw of the medium reached initial values higher than 0.900; for example at the highest production, the Aw value was 0.951. It is interesting to note that in this fermentation, the medium received an inoculum volume two times higher than of rice grits to have a slightly higher water activity. The two media had similar initial water activity, which can be concluded to studying the water adsorption curves.

Comparing the results obtained in the present study with those of previous studies [34], with $B$. thuringiensis israelensis, in which broken rice with high initial moisture content (Ubu $=56.35 \%$ ) was used as substrate, it can be observed that the yield of the present experiment was lower $\left(10^{7}\right.$ spores/g substrate on average versus $10^{8}$ spores/g substrate). The productivity obtained in the fermentation carried out with the rice grits with initial Aw of 0.965 was 1.0 of $\times 10^{6}$ spores/g medium/h, and in the case of the process with broken rice with high moisture content, it was $2.2 \times 10^{6}$. This indicates that there was no significant difference between them, especially considering the difference in the time spent in both experiments. However, the time required for the maximum productivity in those studies was 112 hours, whereas in the present study it was around 36 hours.

Gonzalez et al. [32] showed that the initial moisture content is the most critical fermentation condition because it is related to the activity of water required for microbial growth, and also due to the fact that since the substrate becomes bloated with the pre-treatment, the penetration of microorganisms is facilitated for an effective use of the substrate. Gervais et al. [28], in a study on the fermentation of Penicillium roqueforti in solid medium, concluded that the variations in moisture content did not change the microorganism growth to a given Aw. They also found that for the range of water activity and moisture content studied, the results clearly showed the influence of Aw on the growth of $P$. roqueforti.

These facts emphasize the importance of studying the substrates to be used in solid-state fermentation processes considering their hygroscopic characteristics and also the volume of inoculum to be added because 
depending on the substrate and the microorganism, only the water in the inoculum is sufficient to obtain the Aw necessary for their development. In fact, Loncin [35] found that to a given moisture content, the Aw will vary according to the substrate. Therefore, in the SSF, it is important to know the Aw of the substrate, which can be determined by the sorption isotherms. Grajek and Gervais [17] also found that small changes in the level of Aw led to considerable changes in the substrate moisture. The results obtained in their study prove that Aw can be a limiting parameter in culture development strongly affecting the sporogenesis of $T$. viride and suggesting that Aw values should be monitored throughout the fermentation process.

Analyzing the moisture variations during the cultivations (Figs. 3 and 4), it can be seen that the moisture content of all fermentation processes with low initial Aw increases in the first 12 hours. This is due to the inoculum absorption of water. Moisture declined slightly near the point of greatest production of spores, and in the processes with ground rice grits and bran, the water activity increased with the greatest production. It is important to mention that concerning Aw, only the processes with low initial water activity were monitored because in the other processes with the corresponding increase in water activity in the first hours, these values exceeded 0.970 , which is impossible to determine using this method, considering that the salt with the highest relative humidity used provided an environment with relative humidity of $97 \%$.

According to Murthy et al. [11], the water is redistributed during fermentation process being incorporated into the tissue, which decreases its availability. This decrease in the free water is also due to the fact that there is an increase in the concentration of the solutes given their need to be dissolved and the evaporation of water in the removal of metabolic heat from the substrate, during the formation of reducing sugars, for example. These variations can be estimated

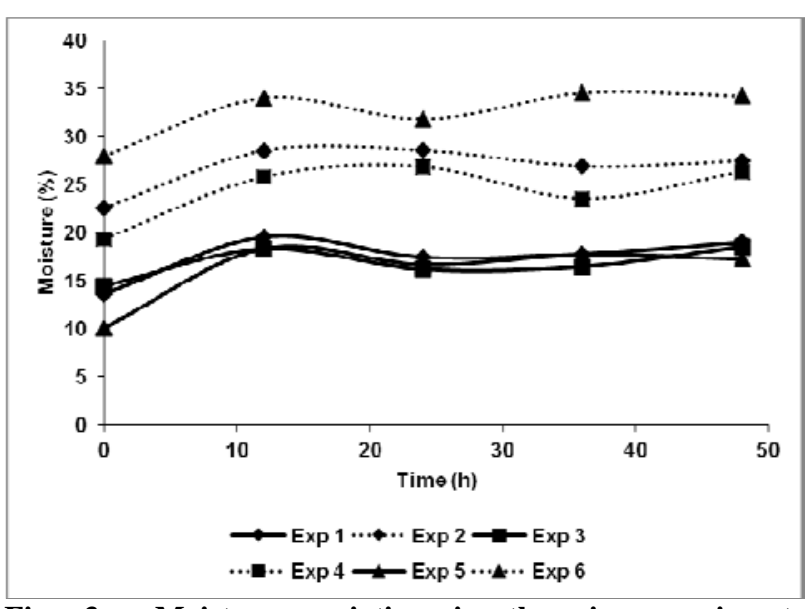

Fig. 3 Moisture variation in the six experiments performed.

Exp 1: rice grits; Awi: 0.620. Exp 2: rice grits; Awi: 0.965. Exp 3: ground rice grits + rice husk; Awi: 0.780. Exp 4: ground rice grits and rice husk; Awi: 0.962. Exp 5: rice bran + rice husk; Awi: 0.615. Exp 6: rice bran + rice husk; Awi: 0.969.

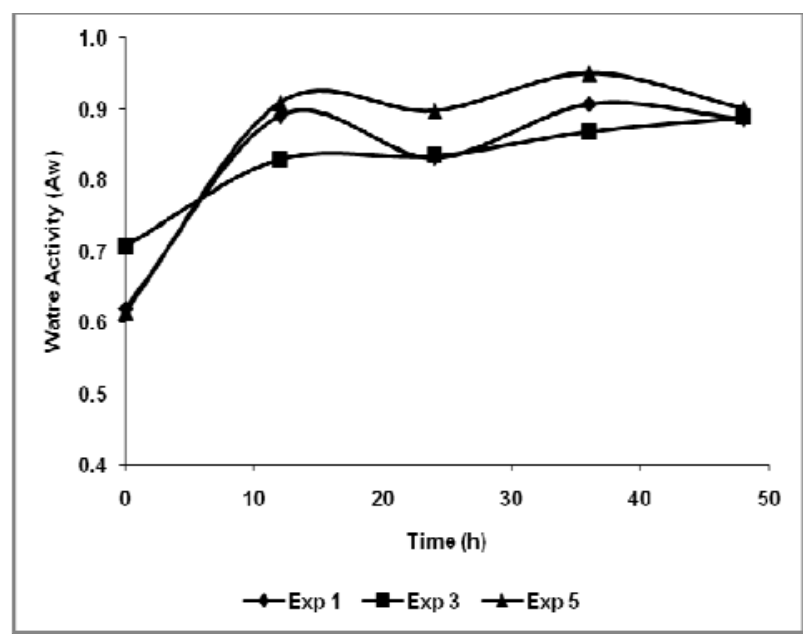

Fig. 4 Water activity (Aw) variation in experiments 1,3 and 5.

Exp 1: rice grits; Awi: 0.620. Exp 3: ground rice grits + rice husk; Awi: 0.780. Exp 5: rice bran + rice husk; Awi: 0.615.

by the method proposed by Ross [36], in which the total Aw is the product of the Aw of each component of the substrate.

Due to the increase in Aw and moisture in the first hours of cultivation, a study was conducted to investigate the influence of water in the inoculums on the culture media, and the results are shown in Fig. 5. $2 \mathrm{~mL}$ of distilled water were added to the culture media, which represents the amount of water present in the inoculum suspension. It can be observed that 


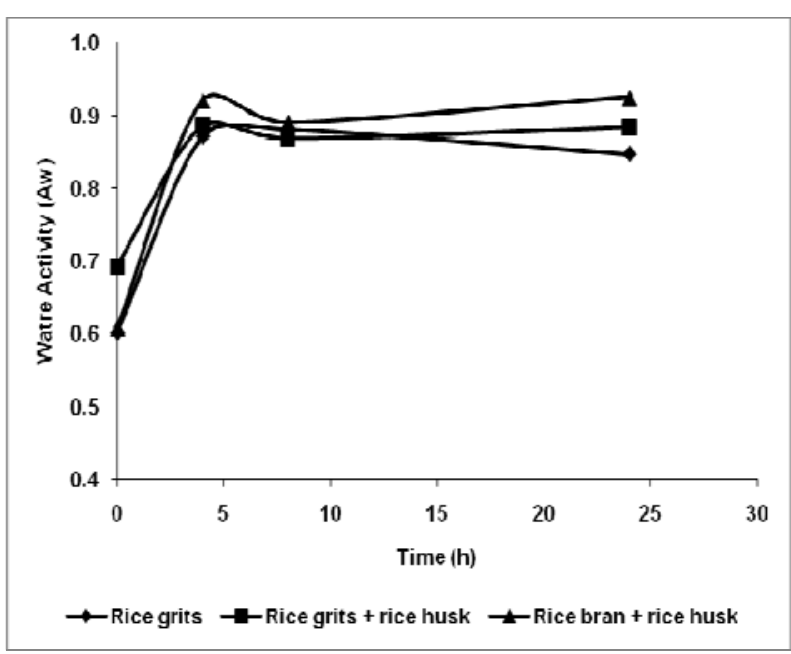

Fig. 5 Water activity (Aw) variation as a function of time after the addition of $2 \mathrm{~mL}$ of distilled water in the propylene bags containing the culture media.

the water added to the culture media is absorbed by the media in the first 4 hours because in this time interval both Aw values and moisture content of all media increase considerably but remain almost constant afterwards.

It can also be noted that the highest increase of Aw took place in the medium composed of bran, in which $2 \mathrm{~mL}$ of water were added in $25 \mathrm{~g}$ of medium, whereas the amount of water added was the same for $50 \mathrm{~g}$ of medium. This might explain why the difference in microbial growth in the fermentations with the bran is lower than that in the fermentations with rice grits. Since bacteria demand more water, they would grow only in the media with high Aw. However, the media with low initial Aw absorb virtually all the water in the first hours of cultivation. Therefore, depending on the volume of the inoculum, in the beginning of the process, the media with low Aw will became with high Aw thus allowing bacterial development.

Another issue to be discussed is that working with lower moisture (sufficient to have an Aw value favorable for microbial growth), the product is obtained in a more concentrated form, which is one of the greatest advantages of solid-state fermentation, which can facilitate the process of extraction and storage. The fermentation product with higher moisture content will undergo a drying process, in which losses can occur and thus, at the end of the process it can have the same or lower concentration of spores than that of the product obtained in this study. It is worth mentioning that costs with energy in the drying process may make the process economically unfeasible. Therefore, the ideal condition is to work with the necessary moisture only to avoid the need to remove excess water, minimize the costs with energy in the recovery of the product, and produce less effluents; concerning the present study, the production time was reduced considerably (from $112 \mathrm{~h}$ to $36 \mathrm{~h}$ ).

\section{Conclusion}

The major conclusion of the present research is that the study of the hygroscopic characteristics of substrates in solid state fermentation is extremely important because depending on the chemical composition of each substrate, or if more than one type of substrate are mixed, the properties of water sorption will be different.

The use of substrates with moisture, i.e., ideal water activity for fermentation proved fundamental, and the excess water does not contribute to the increase in productivity. This means energy saving, effluent reduction, and greater stability.

Rice industry byproducts can be considered as potential substrates for the fermentation process of $B$. thuringiensis. The best results were obtained with the medium composed of rice grits. The productivity ((spores/g medium)/h) for the process with initial Aw of 0.620 was $3.0 \times 10^{4}$, and for the initial Aw of 0.965 , was $1.0 \times 10^{6}$.

\section{References}

[1] M.A. Berbert-Molina, A.M.R. Prata, L.G. Pessanha , M.M. Silveira, Kinetics of Bacillus thuringiensis var. israelensis growth on high glucose concentrations, Journal of Industrial Microbiology and Biotechnolology 35 (2008) 1397-1404.

[2] E.I. Efimtsev, G.P. Burov, O.E. Voronina, Specific features of accumulation kinetics of entomocidal exotoxins of Bacillus thuringiensis Subsp. Thuringiensis during the fermentation stage of bitoxibacillin production, 
Applied Biochemestry and Microbiology 38 (2002) 540-547.

[3] M.E.M. Habib, C.F.S Andrade, Bactérias entomopatogênnicas, in: S.B. Alves (Coord.), Controle Microbiano de Insetos, Manole, São Paulo, 1986, p. 127.

[4] I.O. Moraes, D.M.F. Capalbo, Produção de bactérias entomopatogênicas, in: S.B. Alves (Coord.), Controle Microbiano de Insetos, Manole, São Paulo, 1986, p. 297.

[5] A. Holmberg, R. Sievänen, G. Carlberg, Fermentation of Bacillus thuringiensis for exotoxin production: Process analysis study, Biotechnololgy and Bioengineering 22 (1980) 1707-1724.

[6] M.A. El-Bendary, Production of mosquitocidal Bacillus sphaericus by solid state fermentation using agricultural wastes, World Journal of Microbiology and Biotechnology 26 (2010) 153-159.

[7] P.S. Vimala Devi, T. Ravinder, C. Jaidev, Cost-effective production of Bacillus thuringiensis by solid-state fermentation, Invertebrate Pathology 88 (2005) 163-168.

[8] I.O. Moraes, D.M.F. Capalbo, R.O.M. Arruda, V.L. del-Bianchi, K.R.S. Ascher, Bacillus thuringiensis development from 1971 to 1996: Cases of a research group in Brazil, Israel Journal of Entomology 32 (1998) 45-48.

[9] L.H. Pelizer, Estudo da Influência da Atividade de Água na Fermentação em Estado Sólido de Bacillus thuringiensis, M.S. Thesis, São Paulo University, São Paulo, 1997.

[10] P.S. Crooke, K. Hong, G.W. Malaney, R.D. Tanner, Solid and semi-solid state bioreactors: Static, rotating and fluidized bed fermentors, Journal of Biomass Energy Society of China 10 (1991) 1-17.

[11] M.V.R. Murthy, N.G. Karanth, K. Rao, Biochemical aspects of solid-state fermentation, Advances in Applied Microbiology 38 (1993) 99-144.

[12] A.K.Patel, C.R. Soccol, A. Pandey, Recent advances in solid-state fermentation, Biochemistry Engineering Journal 44 (2009) 13-18.

[13] S.R.B.R. Sella, B.P. Guizelini, L.P.S. Vandenberghe, A.B.P. Medeiros, C.R. Soccol, Bioindicator production with Bacillus atrophaeus' thermal-resistant spores cultivated by solid-state fermentation, Applied Microbiology and Biotechnology 82 (2009) 1019-1026.

[14] S. Zhao, N. Hu, J. Huang, Y. Liang, B. Zhao, High-yield spore production from Bacillus licheniformis by solid state fermentation, Biotechnology Letters 30 (2008) 295-297.

[15] P. Gervais, New sensor allowing continuous water activity measurement of submerged or solid-substrate fermentations, Biotechnology and Bioengineering 33 (1989) 266-271.

[16] J.A. Troller, Water activity and food quality, in: T.M.
Hardman (Ed), Water and Food Quality, Elsevier Applied Science, London, 1989, p. 1.

[17] W. Grajek, P. Gervais, Effect of the sugar-beet pulp water activity on the solid-state culture of Trichoderma viride TS, Applied Microbiology and Biotechnology 26 (1987) 537-541.

[18] B. Hahn-Hägerdal, Water activity: A possible external regulator in biotechnical processes, Enzyme Microbiology and Technology 8 (1986) 322-327.

[19] B.K. Lonsane, N.P. Ghildyal, S. Budiatman, J. Ramakrishna, Engineering aspects of solid state fermentation, Enzyme Microbiology and Technology 7 (1985) 258-265.

[20] W.J. Scott, Water relations of food spoilage microorganisms, Advances on Food Research 7 (1957) 83-127.

[21] W. Horwitz, A. Senzel, H. Reynolds, D.L. Park, Official Methods of Analysis of the Association of Official Analytical Chemists, Association of Official Analytical Chemists, Washington, 1990.

[22] E.C. Bligh, W.J. Dyer, A rapid method of total lipid: Extraction and purification, Canadian Journal of Biochemistry Physiology 37 (1959) 911-917.

[23] O. Zenebon, N.S. Pascuet, P. Tiglea, Métodos Físico-químicos para Análise de Alimentos, Instituto Adolfo Lutz, São Paulo, 2008.

[24] A.H. Landrock, B.E. Proctor, A new graphical interpolation for obtaining humidity equilibria data, with special reference to its role in food packaging studies, Food Technology 5 (1951) 332-337.

[25] P.J. Thompson, K.E. Stevenson, Mesophilic sporeforming aerobes, in: M. Speck (Ed.), Compendium of Methods of Microbiological Examinations of Foods, American Public Health Association, Washington, 1984, p. 211.

[26] G.C. Dors, R.H. Pinto, E. Badiale-Furlong, Influência das condições de parboilização na composição química do arroz, Ciência e Tecnologia de Alimentos 29 (2009) 219-224.

[27] P. Gervais, P. Molin, The role of water in solid-state fermentation, Biochemical Engineering Journal 13 (2003) 85-101.

[28] P. Gervais, M. Bensoussan, W. Grajek, Water activity and water content: Comparative effects on the growth of $P$. roqueforti on solid substrate, Applied Microbiology and Biotechnology 27 (1988) 389-392.

[29] C. Krishna, Solid-state fermentation systems-an overview, Critical Reviews on Biotechnology 25(2005) 1-30.

[30] D.A.A. Mossel, Water relations of foods, in: R.B. Duckworth (Ed.), Food Science and Technology, Academic Press, London, 1975, p. 347. 
[31] L.R. Beuchat, Influence of water activity on esporulation, germination, outgrowth and toxin production, in: R.B. Rockland, L.R. Beuchat (Eds.), Water Activity: Theory and Applications to Food, Marcel Decker, New York, 1987, p. 137.

[32] J.B. Gonzalez, A. Tomasini, G.V. Gonzalez, J. Lopes, Penicillin production by solid state fermentation, Biotechnology Letters 10 (1988) 793-798.

[33] A.Corona, D. Sa'ez, E. Agosin, Effect of water activity on gibberellic acid production by Gibberella fujikuroi under solid-state fermentation conditions, Process
Biochemistry 40 (2005) 2655-2658.

[34] I.O. Moraes, L.H. Pelizer, D.M.F. Capalbo, Estudo da atividade de água e fermentação semi-sólida do Bacillus thuringiensis em arroz, Simpósio Latino Americano de Ciência de Alimentos, Campinas (1995) 37.

[35] M. Loncin, Basic principles of moisture equilibria, in: S. Goldblith, L. Rey (Eds.), Freeze-drying and Advanced Food Technology, Academic Press, New York, 1975, p. 599.

[36] K.D. Ross, Estimation of water activity in intermediate moisture foods, Food Technology 29 (1975) 26-34. 